Artículo

\title{
Una mirada cualitativa al Proyecto de Revitalización del Náhuat
}

\author{
Nahuat Revitalization Project: A qualitative look \\ Ana María Soriano Rodríguez \\ asoriano@udb.edu.sv
}

ISSN 1996-1642, Editorial Universidad Don Bosco, año 9, No.15, enero-junio de 2015, pp. 55-73

Recibido: 15 de diciembre de 2014. Aprobado: 28 de enero de 2015.

\section{Resumen}

Este artículo presenta los hallazgos del estudio sobre la percepción que madres, padres, profesores y estudiantes tienen sobre la revitalización del náhuat y sobre su particular contribución a la revitalización de la lengua moribunda y los aprendizajes dejados en la participación de este tipo de proyectos. El propósito del estudio se enfoca precisamente en obtener una mirada con un enfoque cualitativo y desde los ojos de quienes participan directa e indirectamente del Proyecto de Revitalización del Náhuat que implementa la Universidad Don Bosco desde 2003. Se destaca en el estudio el rol de la comunidad educativa en la enseñanza de una lengua ancestral amenazada y los aprendizajes que contribuyen a una educación que respeta la diversidad cultural y que favorece a la identidad de una sociedad. La investigación se realizó en las escuelas de los municipios de Izalco, San Juan Talpa y Santo Domingo de Guzmán, en El Salvador, con base a veinte y nueve entrevistas enfocadas.

Palabras clave: Multiculturalidad, interculturalidad, idioma, identidad, cultura, náhuat
Abstract:

This paper shows the findings of the research about the perception that mothers, fathers, teachers and students have with regard to Nahuat revitalization, about their particular contribution to the revitalization of an endangered language and about learning as an outcome of their participation in the project. The goal of the study is getting a qualitative look from participants directly and indirectly involved in the Nahuat Revitalization Project carried out by Universidad Don Bosco since 2003. The study highlights the role of the educational community in the teaching of an endangered language, the learning that contribute to an education that values cultural diversity and that favors the identity of a society. The research was carried out in schools of Izalco, San Juan Talpa y Santo Domingo de Guzmán, El Salvador, on the base of twenty-nine focused interviews.

Keywords: Multiculturality, interculturality, language, identity, culture, Nahuat

\footnotetext{
* Profesora investigadora del Instituto de Investigación y Formación Pedagógica de la Universidad Don Bosco y candidata a doctora en educación por la Universidad de Costa Rica.
} 


\section{Introducción}

El Proyecto de Revitalización del Náhuat' ${ }^{1}$ (PRN) inició en el año 2003 con la participación de 3 escuelas y 275 estudiantes. Para el 2008 se había extendido a 11 escuelas públicas ubicadas en zonas de náhuat hablantes y contaba con la participación de más de 2500 estudiante. Para el 2013 el número había ascendido a 40 centros escolares, 5500 niños, niñas y jóvenes, 65 profesores diplomados en Educación Intercultural Bilingüe (EIB) y más de 100 capacitados en lengua náhuat (J. E. Lemus, 2014, comunicación personal, diciembre 18, 2014). Desde que se inició el proyecto contó con un enfoque intercultural con el objetivo de motivar una transformación social, es decir, no se buscaba una enseñanza del náhuat como lengua extranjera, sino que contribuyera a la búsqueda de una sociedad más justa (Lemus, 2008).

Multiculturalidad, interculturalidad, inclusión, atención a la diversidad, son términos que podrían considerarse de uso reciente en el contexto salvadoreño y sus ámbitos educativos. Inclusión y atención a la diversidad son los que han ganado mayor difusión y hacen referencia, por lo general, a la inclusión de personas con alguna discapacidad (sordera, ceguera), a los centros educativos comúnmente referidos con el término de "normales". Hablar de multiculturalidad e interculturalidad pareciera ser innecesario, donde el indígena salvadoreño ha sido invisibilizado y donde los centros de estudios están conformados por grupos de estudiantes cuyas características raciales y socioeconómicas son aparentemente homogéneas.

Los diferentes grupos étnicos de El Salvador son estudiados en las escuelas como parte de la historia. Se enseña en los salones de clase y textos sobre lencas llamados también potones, sobre los cacaoperas y pipiles como parte de la historia de El Salvador, pero muy poco se habla, se enseña o se estudia sobre los grupos indígenas que aún existen y son parte de la realidad actual.

Una de las preguntas que recibo con mayor frecuencia cuando hablo en público sobre la problemática indígena del país es ¿Qué todavía hay indios en El Salvador? y, al contestarles afirmativamente, la siguiente pregunta es ¿Y dónde están? Nadie puede dar cuenta de la existencia de algún grupo indígena en el país, y se sorprenden al decirles que existen al menos tres grupos étnicos distintos (Lemus, 201 1, p. 4).

El informe que presentara el gobierno de turno de El Salvador ante la Convención Internacional sobre la Eliminación de todas las Formas de Discriminación Racial (Naciones Unidas CERD, 2005) -el cual fuera obligado a revisión por la comisiónmanifestaba que la población salvadoreña no está compuesta por grupos con características raciales diferentes, por lo que, consecuentemente se afirmaba que en el país no existía discriminación por motivos de raza. El mismo documento,

Una mirada cualitativa al Proyecto de Revitalización 56. del Náhuat
1. El Proyecto de Revitalización del Náhuat ha sido diseñado y dirigido por Jorge Ernesto Lemus (Ph.D. en Lingüística por la Universidad de Arizona) con el auspicio de la Universidad Don Bosco (UDB). 
afirmaba que en El Salvador se había desarrollado una homogeneidad mestiza, sin mayores diferencias entre blancos e indígenas. El documento no abonaba si está homogeneidad estaba referida a las características físicas, derechos u oportunidades.

Dos años más tarde, a razón del Censo Poblacional de 2007, habitantes de Pushtan, Nahuizalco presentaron una demanda ante la Corte Suprema de Justicia por considerar que el Censo incurría en discriminación racial, ya que reducía la identidad indígena al color de la piel (Gregori, julio, 2007).

El Censo Poblacional 2007 (Ministerio de Economía, 2008) afirma que en El Salvador solamente $0.02 \%$ de la población se reconoce a sí mismo como indígena. Esto tiene causas históricas y socioculturales. El mismo documento afirma que solamente se identificaron 97 náhuat hablantes. No obstante, Lemus (2008), estima que el número es aproximadamente de 200 náhuat hablantes.

No se pretende polemizar sobre las cifras dadas por la Dirección General de Estadística y Censos y los investigadores, sino aclarar que el porcentaje de indígenas en El Salvador constituye una minoría que ha sido invisibilizada por años. Lo que sí se pretende en este artículo es reflexionar, a partir de los datos, sobre el para qué revitalizar una lengua moribunda que contribuye a la identidad y respeto a la multiculturalidad.

Banks (1996) define la educación multicultural (EM) como un campo de estudio y una disciplina emergente cuyo principal objetivo es crear iguales oportunidades para estudiantes de diversas razas, etnias, clases sociales y grupos culturales. El principal objetivo es lograr que los estudiantes adquieran conocimientos, actitudes y habilidades para integrarse efectivamente en una sociedad democrática pluralista para interactuar, negociar y comunicar con personas de grupos diferentes y así, crear una comunidad moral y civil en función del bien común.

Además, según Banks (1996), la EM no puede estar limitada a porcentajes, sino al reconocimiento de esta diversidad cultural, por lo que es válido implementar una revitalización de náhuat en un contexto donde la presencia indígena es mínima. A través de la EM se busca visibilizar a estos grupos y reconocer la herencia cultural en el contexto salvadoreño, especialmente en la zona occidental del país. A pesar de la Masacre de 1932, definida por Lara-Martínez (2008) como el Etnocidio de 1932, la población indígena sigue formando parte tejido social salvadoreño. Lara-Martínez afirma que un genocidio real es favorecido por una ideología racista de una nacionalidad mestiza que por falta de un decreto oficial invisibiliza al indígena y no da espacio al respecto de la diversidad cultural y, por tanto, genera un estereotipo social del indígena. Por lo tanto, la sociedad salvadoreña añade la necesidad de borrarlo del censo oficial, en nombre de una unidad racial mestiza. 
Ante este proyecto auspiciado por la UDB, es válido preguntarse ipara qué revitalizar una lengua moribunda? Se argumenta a su favor, que una educación hacia el reconocimiento de la multiculturalidad, que visibilice al indígena y que borre los estereotipos sociales, no debe limitarse a grupos de estudiantes que forman parte de las minorías, sino más bien, una educación para las mayorías que propicie el respeto y tolerancia a la diversidad y raíces culturales.

Banks (2002) identifica cinco dimensiones de la EM que incluyen: integración de contenidos, construcción de conocimientos, reducción de prejuicios, equidad pedagógica y empoderamiento de las estructuras sociales. El autor reconoce que cada una de las dimensiones está interrelacionada, pero que cada una requiere un enfoque particular. Para el caso salvadoreño, es muy pertinente la dimensión de reducción de prejuicios, tal como Banks lo propone, actividades que ayuden a los estudiantes a desarrollar una actitud positiva hacia las diferentes razas, etnias y culturas, y por tanto, deberían ser consideradas como eje transversal del currículo nacional (García-Castaño, Pulido y Montes, 1997) lo cual indica, sin duda, que la EM significa aprender sobre de los diversos grupos culturales, con especial énfasis en las diferencias y similitudes culturales.

Además, si se busca que la escuela cumpla con su tarea humanista, debe mostrársele a los alumnos que existen otras culturas además de la suya. De allí que, como punto de partida, la escuela tiene que ser local pero tiene que ser internacional e intercultural como punto de llegada (Gadotti, 2003). Esta afirmación concuerda con los planteamientos de Bank (1996) quien propone incluso, tareas sencillas tales como, evitar contar y reírse de bromas relacionadas con grupos culturales y raciales diferentes a los de la cultura dominante, realizar actividades interculturales ya sea de convivencia con otros centros educativos e incluso llevar ayuda a comunidades que los requieran. Son propuestas sencillas que no requieren de grandes inversión económicas, sino la voluntad y respeto de las diferencias

\section{Método}

Esta investigación se realizó bajo el paradigma interpretativo con una metodología de corte cualitativa con una muestra intencionada donde el principal objetivo de la investigación era comprender la percepción que madres, padres, profesores y estudiantes tenían sobre el proyecto, su contribución a la revitalización de la lengua moribunda y los aprendizajes adicionales al participar de este tipo de proyecto. Es decir, tener una mirada del Proyecto de Revitalización del Náhuat (PRN) desde los ojos de quienes participan, directa o indirectamente. No se buscó generar evidencia sobre el dominio del idioma, que correspondería a otros objetivos de investigación y, por tanto, otra metodología.

Una mirada cualitativa al Proyecto de Revitalización

58. del Náhuat

\section{Participantes de la investigación}

La comunidad educativa involucrada en el proceso educativo de la revitalización de la lengua náhuat lo constituyen: 
- Profesores capacitados a través del Diplomado en Educación Intercultural Bilingüe impartido por la Universidad Don Bosco que imparte la clase de náhuat en las escuelas seleccionadas.

- Directores de los centros educativos.

- Padres y madres de familia de alumnos que han estudiado náhuat

- Estudiantes de náhuat de las escuelas que participan del PRN en estudio: (1) Complejo Educativo (C. E) Dr. Mario Calvo Marroquín, Municipio de Izalco, Departamento de Sonsonate; (2) Complejo Educativo Profesor Alberto Varela, Municipio de San Juan Talpa, Departamento de La Paz; (3) Complejo Educativo Santo Domingo de Guzmán, Municipio Santo Domingo de Guzmán, Departamento de Sonsonate. Todos ubicados en El Salvador.

Tabla 1.

Participantes investigación por escuela, municipio, rol y sexo

\begin{tabular}{llllc}
\hline Escuela & Municipio & Rol & Sexo & Total \\
\hline C.E. Profesor Alberto Valera & San Juan Talpa & Estudiante & Femenino & 3 \\
\hline C.E. Profesor Alberto Valera & San Juan Talpa & Maestro & Masculino & 2 \\
\hline C.E. Profesor Alberto Valera & San Juan Talpa & Maestro & Femenino & 2 \\
\hline C.E. Profesor Alberto Valera & San Juan Talpa & Madre de familia & Femenino & 1 \\
\hline C.E. Snto Domingo de Guzmán & Santo Domingo de Guzmán & Estudiante & Femenino & 3 \\
\hline C.E. Snto Domingo de Guzmán & Santo Domingo de Guzmán & Maestro & Masculino & 1 \\
\hline C.E. Snto Domingo de Guzmán & Santo Domingo de Guzmán & Estudiante & Masculino & 1 \\
\hline C.E. Snto Domingo de Guzmán & Santo Domingo de Guzmán & Madre de familia & Femenino & 1 \\
\hline C.E. Snto Domingo de Guzmán & Santo Domingo de Guzmán & Padre de familia & Masculino & 1 \\
\hline C.E. Dr. Mario Calvo Marroquín & Izalco & Estudiante & Femenino & 4 \\
\hline C.E. Dr. Mario Calvo Marroquín & Izalco & Madre de familia & Femenino & 2 \\
\hline C.E. Dr. Mario Calvo Marroquín & Izalco & Maestro & Masculino & 1 \\
\hline C.E. Dr. Mario Calvo Marroquín & Izalco & Estudiante & Masculino & 1 \\
\hline Cuna Náhuat & Santo Domingo de Guzmán & Madre de familia & Femenino & 5 \\
\hline Cuna Náhuat & Santo Domingo de Guzmán & Padre de familia & Masculino & 1 \\
\hline Total entrevistados & & & $\mathbf{2 9}$ \\
\hline
\end{tabular}

\section{Recolección de datos}

La recolección de datos se realizó a través de la técnica de entrevista enfocada (Ruiz Olabuenaga e Ispizua, 1989) y con la ayuda de una guía de preguntas generadoras como instrumento. Dichas entrevistas fueron realizadas, en el caso de los profesores, directores y estudiantes en las escuelas respectivas y en cuanto a las madres y al padre de familia en su residencia. En total, se realizaron 29 entrevistas que fueron grabadas, bajo consentimiento informado de los participantes y posteriormente transcritas. 
Tal como lo sugiere Creswell (2009), se requiere de 20 a 30 entrevistas para llegar a una saturación de los datos. Por lo que, se realizaron al menos 20 entrevistas, mientras simultáneamente se analizaron datos. Además, para considerar el número total de entrevistas se tomó el criterio que establece Corbin y Strauss (2008) con respecto a la saturación de los datos en el sentido que esta se ha alcanzado cuando ya no emergen nuevas categorías o temas, pero además, cuando cada categoría establecida se ha explorado en profundidad en cuanto a sus dimensiones y propiedades y están suficientemente desarrolladas en coherencia con los objetivos y planteamiento del problema de la investigación.

\section{Procesamiento de los datos}

A partir de la recolección y transcripción de las entrevistas se inició con la codificación. Debe recordarse que un código es un nombre o símbolo para representar e identificar ítems, ideas o fenómenos que el investigador ha advertido en los datos. Usualmente a un código se le asigna un nombre o frase corta, el cual representa la abstracción (no descripción, ni inferencia) de los datos (LeCompte y Schensul, 1999).

Para establecer los códigos, antecedió la identificación de incidentes, es decir, cada parte de la unidad de muestreo que pueda ser considerada como analizable separadamente o porque aparece en ella una de las referencias que el investigador considera importante. Dichos fragmentos del contenido fueron aislados y separados por aparecer allí uno de los símbolos, palabras clave o temas que se consideran oportunos desde los propios datos (Requena, Carrero y Soriano, 2006).

Posteriormente, se procedió con una codificación abierta (Corbin y Strauss, 2007) para generar categorías iniciales y se describieron sus propiedades. Este tipo de codificación es denominada abierta dado que, para desarrollar los conceptos se abre el texto y se exponen pensamientos, ideas y significados contenidos en él. Los datos se descompusieron discretamente, se examinaron y se compararon similitudes y diferencias. Cuando los códigos se acumularon se inició un proceso de categorización, las categorías fueron construidas en función de sus propiedades y dimensiones. Las propiedades son las características generales de una categoría, mientras que las dimensiones representan la localización de una propiedad en un continuo o rango.

Todos los datos transcritos fueron procesados con asistencia del software para el análisis de datos cualitativos NVIVO 10, el cual facilita la organización de códigos, categorías, procesamientos de gráficas, árbol de palabras y mapa de relaciones categorías (nodo)-entrevistados (recursos).

Una mirada cualitativa al Proyecto de Revitalización

60. del Náhuat
A continuación se etiquetaron las diferentes codificaciones identificadas y abstraídas a través del proceso de análisis de las entrevistas, se explica cómo se entienden cada una de ellas: 
- Beneficios educativos: beneficios que favorecen los procesos cognitivos.

- Beneficios identidad: beneficios que advierten los participantes de la investigación en función de fortalecer la identidad, la herencia cultural.

- Beneficios sociales: que beneficios identifican los participantes de la investigación en relación al reconocimiento social que obtienen dado al aprendizaje (estudiantes, madres y padres de familia) o enseñanza del náhuat (profesores, directores)

- Rol abuelos: Acciones de los abuelos y abuelas que contribuyen a la práctica del náhuat y a fortalecer la relación abuelos-nietos.

- Rol docente: Acciones del profesor en el aula y el proceso de enseñanzaaprendizaje del idioma. Motivación al estudiante.

- Rol estudiante: Función de los estudiantes en el proceso de revitalización.

- Rol padre, madre: acciones (motivación, facilitar recursos, acompañamiento) de los padres y madres de familia para que su hijo o hija aprenda el idioma.

- Reconocimiento idioma: discurso del entrevistado en el cual se reconoce al náhuat como idioma y no como dialecto.

- Salto generacional: concreciones en el discurso de los entrevistados que evidencia el salto generacional del aprendizaje del náhuat, (los ahora abuelos no trasmitieron el idioma a sus hijos y quienes en la actualidad aprenden son los nietos o nietas)

\section{Resultados}

El proyecto de revitalización del náhuat se propuso desde sus inicios (2003) cinco componentes que favorecen y revalorizan la cultura: 1) identidad cultural, 2) corpus lingüístico, 3) legislación, 4) educación y 5) bienestar social. Siendo el primero, identidad cultural, el de mayor importancia ya que éste impulsa a los demás. A su vez, la identidad cultural, está íntimamente ligada a la lengua, por

\section{Diagrama 1.}

Componentes del proceso de revitalización lingüística (Tomado de Lemus, 2004, p. 26).

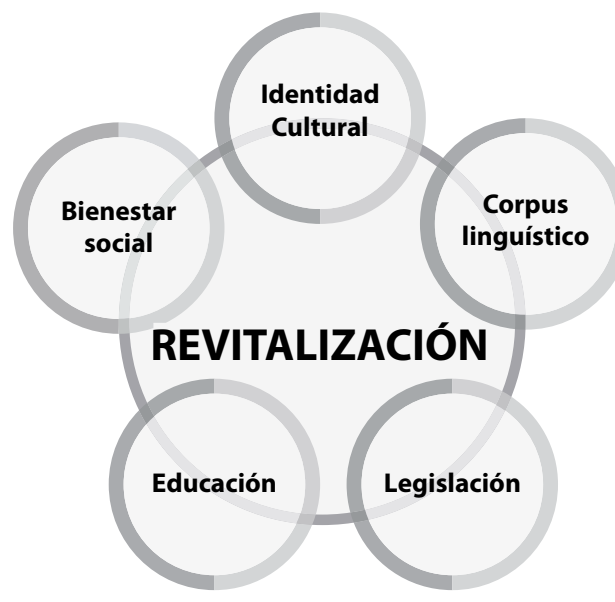


lo que su pérdida implica un peligro inmediato. En el caso del náhuat, dado la pérdida de hablantes y pérdida de la identidad cultural son necesarios esfuerzos que contribuyan a revivirla y motivar el deseo de hablarlo. (Lemus, 2008).

A través de esta investigación se ha evidenciado que las dimensiones más fortalecidas con el proyecto han sido la identidad cultural, bienestar social y educación. Los resultados de las entrevistas han establecido la tendencia que los participantes del proyecto al aprender el náhuat con lo cual contribuye a generar y reconocer la identidad cultural perdida o desconocida.

El bienestar social ha sido otra de las dimensiones que se han evidenciado a través de los datos cualitativos, aunque debe especificarse que este bienestar social, tal como lo define Lemus (2008), implica también un mejoramiento económico, lo cual no ha sido evidente en todas las personas entrevistadas; sin embargo, sí se identifica, como se ha denominado en esta investigación, un beneficio social, en cuanto que, el aprender y enseñar náhuat conlleva un reconocimiento y distinción entre los profesores y estudiantes (ver Ref. 01) que no estudian ni imparten este idioma.

$\begin{array}{lll}\text { Ref. } 01 \text { El beneficio es más personal, ya si hay un beneficio } & \text { Profesor 04, } \\ \text { económico y material, eso ya va ser ganancia; } & \text { C.E. Dr. Mario Calvo } \\ \text { pero personal en el sentido que nos diferencia de } & \text { Marroquín,- Izalco, } \\ \text { los demás. Cuántas veces hemos tenido visitas de } & \text { Sonsonate. } \\ \text { personas que nos hablan de sus costumbres, de sus } & \\ \text { idiomas y nosotros nos quedamos callados, ipor qué? } & \\ \text { Porque no sabemos, porque desconocemos nuestra } & \\ \text { identidad, vale la pena para darnos a conocer al } & \\ \text { exterior lo que somos, lo que nos pertenece. } & \end{array}$

Estas tres dimensiones: identidad cultural, bienestar social y educación están relacionadas entre sí, a través de las entrevistas y el proceso de codificación. Asimismo, se identificaron varios fragmentos, en los que de forma individual bien podrían incluir las tres dimensiones (ver Ref. 01).

\section{a. Contribución a la identidad salvadoreña}

De acuerdo con Lafaye (1986), la identidad cultural latinoamericana se ha caracterizado por un desgarramiento interno y ambivalencia externa. Reconoce que la identidad cultural es por hoy un devenir, un proceso en vía de desarrollo, una gestación vital. Para Taylor (1996), el discurso de la identidad es complejo, ya que constituye un concepto psicológico con el cual se busca comprender una dimensión de la conciencia de sí mismo. La identidad es lo que sitúa a la persona en el mundo real y para que sea propia del ser humano debe ser aceptada, lo que implica una negociación con el entorno, la historia y el

Una mirada cualitativa al Proyecto de Revitalización 62. del Náhuat destino. Mientras no se reconoce como parte original de la persona, no podrá reclamarse propia, por tanto, debe en principio ser asumida. 
Es en este proceso de reconocimiento y aceptación de la identidad, que el proyecto de revitalización ha contribuido directamente. Como ya se explicó anteriormente, el objetivo de esta investigación no fue la medición de conocimientos o aprendizajes; sino, la perspectiva cualitativa que los participantes tienen el sobre proyecto. Los entrevistados reconocen como el respeto por los antepasados, el orgullo de ser salvadoreño lo demuestra por su conocimiento del náhuat, reconocer sus raíces, respeto al otro y evitar la alienación e imitación de otras culturas (ver Ref. 2-9). Así, se encontró a través de las 29 entrevistas realizadas, se encontró que en al menos 24, se identificaron 50 referencias relacionadas con el interés del estudio del náhuat en función de favorecer la identidad y herencia cultural, tanto en los estudiantes como en maestros y madres de familia.

La figura representa a los entrevistados (24 de 29) los cuales mencionaron que el aprendizaje del náhuat contribuye a la identidad cultural. Las palabras clave involucran pasado, provenimos, historia, cultura, reconocer y otras similares.

Ref. 02 Es una oportunidad para conocer lo que fue el pasado en nuestro país, lo que hablaban ellos, es una gran oportunidad, Me gusta, porque aprendo muchas cosas, porque tenemos que aprender que fue nuestra historia y tenemos que rescatar, porque es algo propio de nuestro país.

Ref. 03 Para mí está bien, porque es algo de nuestros antepasados y es algo que ya está en decadencia y me parece bien que estemos tratando de recuperarlo, practicarlo más.

Ref. 04 Primero porque siento, como dicen es un bien intangible pero valioso para la historia de nuestro país, el rescatar la lengua de nuestro país, es una reliquia aquí, porque ya somos muy pocos pueblos, donde personas hablan Nahuat, y la verdad que es un idioma muy bonito que a cualquiera cuando ya lo aprende le siente sabor al hablar el idioma.

Ref. 05 Es importante porque es nuestras raíces, de allí provenimos y necesitamos reconocer de dónde venimos y debemos tener presente eso.

Ref. 06 A mí me gusta bastante el náhuat ya que es un idioma propio de nosotros y que la verdad es importante aprenderlo y no debemos avergonzarnos de esto así como muchos que se avergonzaban del náhuat, pero no, el nábuat es nuestras raíces y aunque algunos digan que es aburrido, es mentira porque es bonito aprender, porque nos puede servir para muchas cosas, tal vez así, este es lindo aprender sobre nuestros antepasados y no tenerlos de menos.

Yo tengo mis sueños, verdad y quisiera irme a estudiar fuera del país y quisiera que vean que de veras soy salvadoreña, hablando mi idioma en náhuat, si alguien me preguntara si soy de El Salvador, si yo pueda decir si con orgullo, y si me preguntaran que has aprendido allá, mi lengua nativa.
Alumna 01, 12 años,

C.E .Dr. Mario Calvo

Marroquín, Izalco,

Dpto. Sonsonate.

Alumna 03, 15 años, C.E. Prof. Alberto Varela, San Juan Talpa, Dpto. La $\mathrm{Paz}$.

Director 01.

Alumna 07, 15 años, C.E. Prof. Alberto Varela, San Juan Talpa, Dpto. La Paz.

Alumna 09, 13 años, C.E. Dr. Mario Calvo Marroquín, Izalco, Dpto. Sonsonate. 
Ref. 07 Yo pienso de que como fue un idioma de los antepasados, que es un idioma propio aquí de El Salvador, para que no se pierda esa costumbre o cultura de nuestros antepasados, porque aquí en este pueblo no, no se habla náhuat, solo los niños estos que estudian aquí y la institución del departamento de La Paz es la única que habla náhuat, es de beneficio para los niños.

Ref. 08 En el mundo globalizado en que estamos ahora, en la tecnología que nos bombardea, pues si uno logra captar, reconocer de dónde viene, quien es uno, verdad, cuáles son sus raíces históricas, uno no es persona alienada, enajenada de este mundo, verdad, que se deja llevar por otras formas de cultura que existen en el mundo, sino que uno valora la de uno, uno no se deja influenciar por otra.

Ref. 09 Porque mira aquí, el color autóctono (se refiere al color de piel de los niños de la escuela). La importancia es que el (estudiante) reconozco a sus antepasados, sus raíces, que de allí procedemos, y ellos en sus rostros, es que cabal lo original, lo que era pipil, maya, que sepan que tenían un idioma, que no hablaban con señas, que eran inteligentes.
Mamá 02,

C.E. Prof. Alberto Valera, San Juan Talpa,

Dpto. La Paz.

Profesor 02,

Prof. Alberto Valera,

San Juan Talpa,

Dpto. La Paz.

\section{Profesor 07}

C.E. Dr. Mario Calvo

Marroquín, Izalco, Dpto. Sonsonate.

\section{Diagrama 2.}

Referencias e identidades según entrevistados.

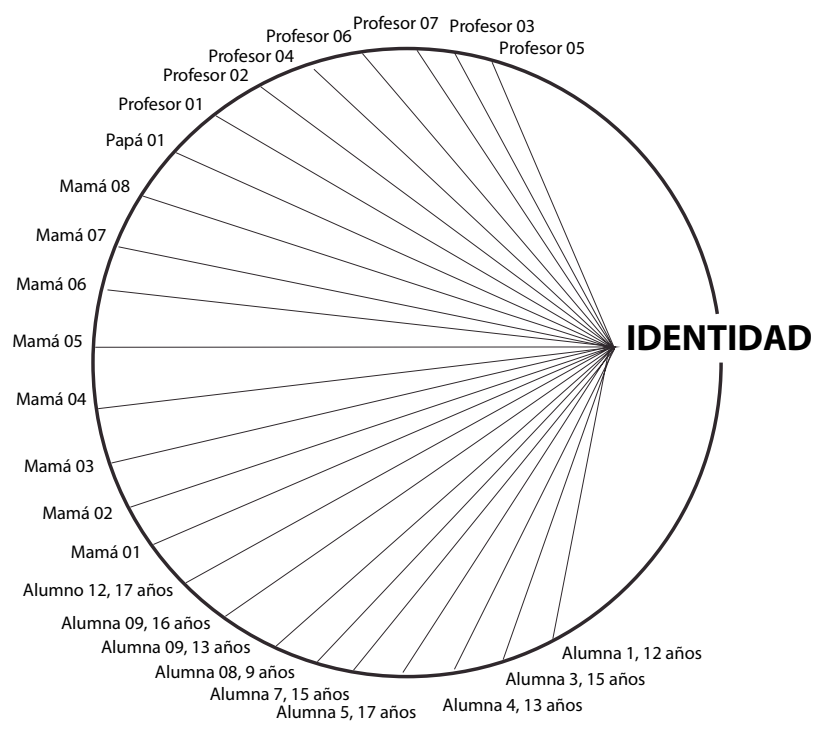

Una mirada cualitativa al Proyecto de Revitalización 64. del Náhuat

\section{b. Nahuat reconocido como un idioma}

De las 29 entrevistas realizadas, en 22 se identificaron 87 referencias que identifican al náhuat como un idioma. Este hallazgo fue alentador dado que comúnmente se le confunde con un dialecto. El árbol del palabras (ver Figura No.1) permite identificar que la palabra idioma en el contexto de las entrevistas, está en función 
del náhuat y se evita asumir que la palabra fuese mencionada aisladamente en relación a otro tema u otro idioma. (Ver Ref. 10-15 y Figura No. 1).

\section{c. Madres, abuelos, profesores y estudiantes}

Tal como lo estable Lemus (2008), padres y madres de familia y la comunidad, en general, son participantes necesarios en este proceso para la transformación social. Además, la EM centrada en la escuela, ha implicado cambios en los paradigmas de enseñanza y percepción de los maestros hacia el náhuat, la estrategias didácticas y la motivación hacia sus estudiantes (Ver Ref. 16, 18, 19, 20).

Ref. 10 Que se empiece a rescatar ese idioma porque es propio de nuestro país.t

Ref. 11 Eh, Nos puede servir para muchas cosas porque en nuestro país todavía existen, al encontrarnos con esas personas, nos ayudaría mucho el idioma.

Ref.12 Es bien importante, porque es algo no sé, es bonito conservar el idioma y que las demás personas vean el orgullo de nosotros, todavía seguir hablando ese idioma, no perderlo, seguir practicándolo.

Ref. 13 La verdad que es un idioma muy bonito que a cualquiera, cuando ya lo aprende le siente sabor al hablar el idioma.

Ref. 14 Mire, yo pienso que quizás un poco quizás si porque, como ese idioma se ha perdido por completo, porque ya la gente no habla, allá en la escuela es donde se les está dando clases así.

Ref. 15 (...)nos hemos sentido satisfechos, por el hecho de enseñar el idioma.
Alumna 01, 12 años, C.E. Dr. Mario Calvo Marroquín, Izalco, Dpto. Sonsonate

Alumna 03, 15 años, C.E. Prof. Alberto Varela, San Juan Talpa, Dpto. La Paz

Alumna 04, 13 años C.E. Prof. Alberto Varela, San Juan Talpa, Dpto. La Paz

Director 01

Mamá 05,

Cuna Náhuat, Santo Domingo de Guzmán, Dpto. Sonsonate

Profesor 04,

C.E.Dr. Mario Calvo Marroquín, Izalco,

Sonsonate.

La motivación por parte de los padres y madres hacia los estudiantes es vital para el aprendizaje, particularmente porque afrontan crítica del para qué aprender una lengua moribunda y no otra, como el inglés. (Ver Ref 17, 18, 20, 21)

Obviamente, los actores principales de este proceso son los estudiantes, son ellos quienes llevan la responsabilidad de revitalizar el idioma como resultado de su aprendizaje. (Ver Ref. 18)

El diagrama representa a los entrevistados (26 de 29) que reconocieron la contribución de un trabajo conjunto por parte de toda la comunidad educativa (padres, madres, abuelos, profesores, estudiantes), predomina, sin embargo, la labor de los padres y madres de familia en la educación de los hijos.

\section{d. Beneficios sociales y educativos}

De acuerdo con las dimensiones establecidas por Lemus (2008), el bienestar social está comprendido con el bienestar económico y educativo de una 
Ref. 16 (Rol profesor) Es algo dinámica, en el aspecto que el profesor pregunta por medio de cualquier juego, pone canciones en náhuat, entre todos comenzamos a traducir. Hacemos actividades grupales, dibujos. De vez en cuando tanto también cantamos.

Ref.17 (Rol mamá) Para que tenga éxito, uno de mamá tiene que apoyar a los hijos para que siempre sigan adelante con esas clases, motivarlo. Yo le doy ánimos, si tiene deberes hágalo, si quiere aprender, aprenda lo que ud. quiera, solo no lo dejamos salir en grupo, es lo único.

Ref.18 (Rol estudiante, profesora, idioma, identidad) La edad que los niños están son como esponjitas, fijese, no necesariamente tienen que saber leer y escribir para saber un idioma, porque así como nosotros lo hacemos se le queda bastante y como lo hacemos bastante creativo, eso facilita que los niños tengan bastante vocabulario de lo que es náhuat y consideramos que es bien importante no para decirle que van hablar 100\% náhuat, pero al menos para decirle, que lo conozcan, que conozcan las raíces. Por el momento se les está dando así, glosario, cancioncitas y según vayan ellos creciendo van ir a conociendo más a fondo lo que es idioma náhuat

Ref. 19 (Rol profesor) Por ratos me dan ganas de desistir, pero me he puesto una meta, voy a hacer como los bancos, cuando uno no adquiere un producto, le buscan lado para ofrecerle otro producto, de esa manera estamos aquí con bachillerato, hemos tenido problemas, pero a la vez, a la misma le buscamos solución, para que los alumnos siempre reciban el náhuat de cualquier forma, allí estamos ya con otra compañera que nos va ayudar, para que los dos segundos años de bachillerato también reciban náhuat

Ref. 20 (Rol mamá) Con mi mamá, hacemos cuestionarios y dice que escriba de español a náhuat (Rol profesor). El nos dice que tenemos que aprender lo que es nuestro país, y nos inspira a aprender cosas nuevas.

Ref. 21 Es bastante importante apoyarlo, yo he oído comentarios, "no para que van a aprender eso", "si eso ya quedó atrás, ya es pasado, que aprendan otras lenguas, el español, el inglés", entonces, allí es bien importante el apoyo de la mamá, el papá, porque digo yo, sino ellos se desmotivan y no le ponen interés a la clase, vea.
Alumno 12, 13 años, C. E. Dr. Mario Calvo Marroquín, Izalco, Dpto. Sonsonate

Mamá 08

C. E. Dr. Mario Calvo Marroquín, Izalco, Dpto. Sonsonate

Profesora 05

C. E. Prof. Alberto Varela, San Juan Talpa, Dpto. La Paz

Profesor 06

C. E. Santo Domingo de Guzmán, Municipio Santo Domingo de Guzmán, Sonsonate

Alumna 10, 8 años C. E. Dr. Mario Calvo Marroquín, Izalco, Sonsonate

Mamá 07

C. E. Dr. Mario Calvo Marroquín, Izalco, Sonsonate
Una mirada cualitativa al Proyecto de Revitalización 66. del Náhuat 


\section{Diagrama 3.}

Referencias a la función de acuerdo con el rol de los entrevistados

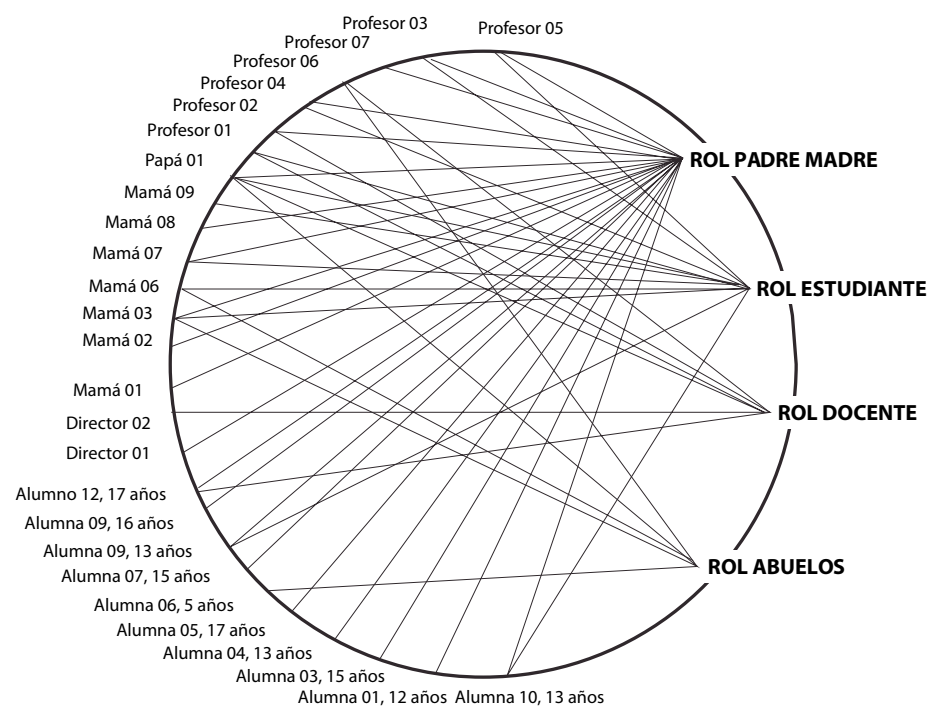

educación que favorezca la identidad cultural. Según las entrevistas realizadas, los participantes no reconocen directamente un beneficio económico inmediato, sin embargo, si atribuyen al hecho de aprender y enseñar el náhuat como un factor que los posiciona dentro de la sociedad y les permite sobresalir entre las demás personas (ver Ref.23, 24, 25). Las madres entrevistadas expresan que ellas y sus hijos e hijas advierten una oportunidad a futuro, particularmente como agentes multiplicadores del náhuat (ver Ref 22, 26, 27).

Por otra parte, los beneficios educativos entendidos en esta investigación como los aprendizajes adicionales al aprendizaje del náhuat en sí, reconocen que aprender un segundo idioma favorece los procesos cognitivos (ver Ref. 28)

El diagrama representa a los entrevistados (23 de 29) que reconocen los beneficios, principalmente los denominados para los fines de esta investigación, beneficios sociales y educativos.

\section{e. Integración de tres generaciones y salto generacional}

Las entrevistas evidencian que el proyecto ha contribuido a generar lazos entre hijos, hijas-madres, padres-abuelo, abuela, (Ver Ref. 31, 33), tal como lo expresa Lemus (2008). Los nietos y bisnietos de la última generación son el objetivo del proyecto y dado que aun conviven en las comunidades, los más niños y niñas mantienen una relación con los ancianas náhuat-hablantes y, por tanto pueden tener la oportunidad de practicar el idioma. Además, los entrevistados reconocen ese salto generacional que provocó la pérdida de la lengua (Ref $29,30,32)$, los padres no enseñaron a sus hijos y quienes ahora aprenden son los 
Ref. 22 Yo pienso que hasta quizás puedan encontrar un empleo, porque, va allí, está una señora que veo que de esto está trabajando del rescatamiento y obtiene ingresos, fuera bueno porque, va un muchacho trabaja en eso, ipor qué está ganando él?, porque puede ese, (calla) ese habla. Yo pienso, que tal vez alguien de estos niños pueda llegar aprender y puedan tener un lugar para que también ellos puedan ganarse la vida de esa manera fuera bueno. Mañana como no sabemos, pero es bueno prepararlos para que también ellos, este, vayan creciendo y aprendiendo y les va a beneficiar mucho a ellos, yo así pienso.

Ref. 23 Los motiva a salir adelante, los sacan, como hay un grupo, lo sacan, a varios lugares a cantar, entones siento les ayuda bastante, yo no puedo nada, pero él sí, siento que habla un poquito, una clase, está bonito, ya que nosotros cuando estudiamos no nos enseñaron nada de eso verdad, nadie nos enseñó y ya que ellos les están enseñando es bonito que apriendan otra lengua, ¿verdá?.

Ref. 24 Soy más reconocido en la comunidad por el hecho que enseño náhuat. He llegado a conocer personalidades gracias al náhuat, como si hoy en día el náhuat, lo leí, como si está de moda. Tenemos la experiencia que fuimos a visitar al viceministro de educación, cuando llegamos allí, nos empiezan a hacer muchas preguntas, "que quienes somos, a qué llegamos, permítame vamos a averiguar" y no sé cómo se me ocurrió y les digo, "somos los maestros de náhuat", "ah porque no me dijeron antes, ipasen, pasen!", todo eso a uno lo hace sentirse bien, hemos compartido momentos con el presidente, nos ha servido para conocer lugares del país que no conocíamos.

Ref. 25 Cuando dicen, qué especialidad tiene y cuando dicen el idioma inglés nadie se extraña, cuando dicen el idioma náhuat, toda la gente ya quiere que le diga unas cuatro palabras en nahuat, que si a donde se imparte y es aquello que se siente la diferencia no es común, y también como muchas personas como le dije al principio desconocen ese idioma

Ref. 26 Nunca sabemos el mañana, eh, Dios prepare un futuro nuevo para ella y llegue ser alguien en el mañana y sea necesario que pueda, aprender y después enseñar ella.

Ref. 27 Yo digo que le va ayudar, digamos porque allí está aprendiendo el idioma y tal vez en un futuro él siempre se va acordar de las palabras y como yo veo si a él le gusta eso, puede tener un trabajo de profesor si al él le gusta y como uno no sabe las ideologías de ellos, como, que es lo que van a pensar después, pero está bonito, porque va aprender más que yo.
Mamá 03

Cuna náhuat Municipio

Santo Domingo de

Guzmán, Dpto. Sonsonate

Mamá 08

C. E. Dr. Mario Calvo

Marroquín, Izalco, Dpto.

Sonsonate

Profesor 04

C. E. Dr. Mario Calvo

Marroquín, Izalco, Dpto.

Sonsonate.

Profesora 03

C. E. Prof. Alberto Varela, San Juan Talpa, Dpto.

La Paz

Mamá 05

Cuna náhuat Municipio

Santo Domingo de

Guzmán, Dpto. Sonsonate

Mamá 01

Cuna náhuat Municipio

Santo Domingo de

Guzmán, Dpto. Sonsonate
Una mirada cualitativa al Proyecto de Revitalización 68. del Náhuat 
Ref. 28 Nada menos hoy, este día, le comentaba a mi compañera que ya hay 4 niños que ya aprendieron a leer y yo me he admirado y es cierto lo que dicen los estudios que un niño que está aprendiendo un segundo idioma, es que no le cuesta aprender lo demás, porque ya puede la lección de mamá y a la vez puede comunicarse en el idioma entonces, eso lo estamos haciendo nosotros, llevando las dos cosas, impartiendo el idioma y enseñándoles ya, preparándolos ya para que vayan a primer grado.

Ref. 29 Mi papá podía, pero él nunca nos habló así. Hemos aprendido palabritas, pero yo, porque otras señoras oigo que hablan, pero él nunca nos enseñó, y por eso es que se ha perdido bastante, porque ya una conversación larga, ya uno no la entiende. Y ellos bien platican y fuera bueno que los niños aprendieran bastante para que así, pues no se terminara digamos esa habla.

Ref. 30 Mi abuela puede, mi suegra también puede, mi mamá puede, pero ella solo lo puede entender, escribirlo, pronunciarlo, no puede, ella solo entiende cuando otras personas hablan en náhuat, ella bien entiende lo que dicen, pero ella pronunciarlo, no puede.

Ref. 31 Bueno, la mayoría alegres, incluso los niñitos que hablan en su casa se conectan con los abuelos y sus abuelos les ayudan allí en las tareas.

Ref. 32 Bueno, (calla por unos segundos) mi papá y mi mamá eran nahuablantes, de ellos aprendí poco, entre ellos hablaban, pero yo poco lo entendí, luego a través de capacitaciones y también así, por esfuerzo personal he ido aprendiendo, pues no mucho, pero digo que ya domino un poco.

Ref. 33 Vea, quizás dicen, yo sé y mi mami no sabe, entonces ellos se ríen y a la vez ellos mismos me dan la respuesta, así, entonces, como motivarles, así, ellos me dicen, "mami y ud nunca vio nahuat y isus abuelos?" No sé, no los conocí, pero ella me dice, porque es importante, es importante para que sepas como hablaban tus antepasados, porque los antepasados de uno así se entendían. Incluso me dice las palabras que decimos, pero no sabemos que es náhuat, el elote por ejemplo, ella me dice, "fíjate" me dice, "de que vos hablas en náhuat", y "porque" le digo yo, "mira decís palabras que n sabes que son en náhuat y las decís, ¿Cuáles son?, el elote, el chilacayote", parece que me dijo, "el tamal", esas cosas así, esas son palabras en náhuat y vos ni cuenta te das que las decís, así es que le digo, ellos están bien motivados con esto del lenguaje que le están enseñando.
Profesora 03

C. E. Prof. Alberto Valera, San Juan Talpa, Dpto.La Paz

Mamá 03

cuna náhuat Municipio

Santo Domingo de

Guzmán, Dpto. Sonsonate

Mamá 06

Cuna náhuat Municipio

Santo Domingo de

Guzmán, Dpto. Sonsonate

Profesor 06

C.E Santo Domingo de Guzmán, Municipio Santo Domingo de Guzmán, Dpto. Sonsonate

Mama 07

C.E. Dr. Mario Calvo Marroquín, Izalco, Dpto. Sonsonate 


\section{Diagrama 4.}

Referencias a la función de acuerdo con los entrevistados.

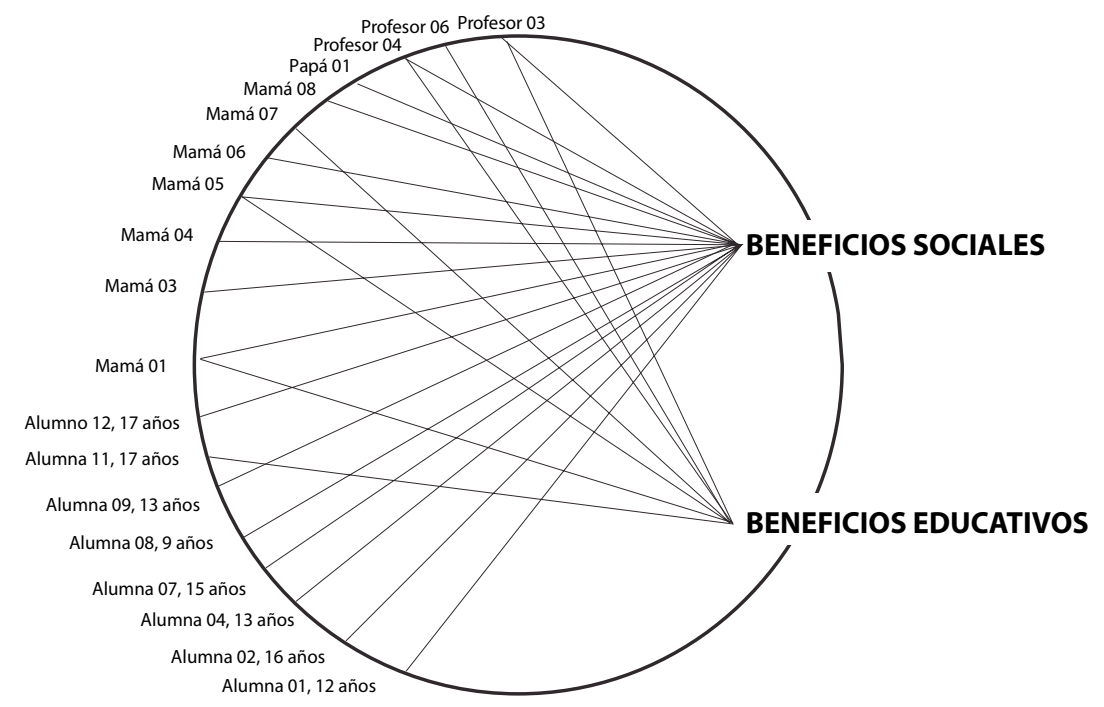

nietos, nietas y practican con abuelo, abuela, especialmente, en el Municipio de Santo Domingo de Guzmán donde se da aún la presencia de hablantes nativos del náhuat.

\section{A manera de reflexión final}

Una educación multicultural debe contribuir a una sociedad respetuosa, tolerante y que fomente una cultura de paz tan necesaria en El Salvador. Para ello, estos grupos minoritarios, aunque no evidentes en todos los salones de clases, deben ser visualizados en las aulas mismas, es decir, educar hacia el respeto que debe existir hacia la diversidad cultural y nuestras raíces culturales. Lemus (2008) sostiene que, dado que los estudiantes dentro del proyecto son parte de la cultura dominante, se busca generar la tolerancia, comprensión y respeto a grupos excluidos por la sociedad. Además, reconocer los valores indígenas y comprender las diferencias culturales dentro de su propia comunidad.

La Educación Multicultural e Intercultural recae, en primera instancia en un cambio de paradigma en la formación de un docente que se empodere de estas teorías y encuentre su aplicabilidad desde el ámbito asignado. Ya sea por decisión propia o porque los azares del destino lo han llevado hasta allí. La EM, especialmente en El Salvador, no debe entenderse exclusivamente como educar en y para contextos multiculturales, sino educar en primera instancia hacia el respeto de las diferentes culturas a través de su visualización, ya sea que se tenga contacto directo con éstas en la cotidianeidad o solo eventualmente.

Una mirada cualitativa al Proyecto de Revitalización

70. del Náhuat
Solamente cuando se dé ese reconocimiento a la multiculturalidad se podrá trascender hacia la interculturalidad. Mientras que multiculturalidad se refiere 
a esa coexistencia de diferentes grupos culturales en un mismo espacio, la interculturalidad trasciende a la interacción y respecto con el otro. Además debe tenerse en cuenta que intercultural no se da de por sí en un centro educativo que reúne a representantes de diversas culturas sino en espacios y entre personas que están dispuestas a aprender y enseñar desde y para la interculturalidad, o sea, en ambientes interculturales de aprendizaje (Meléndez, 2008). Comprendida la educación intercultural bajo estas premisas, esta lograría tener impacto en ambientes educativos cuya diversidad étnica es homogénea, pero que es capaz de advertir la heterogeneidad de cada uno de sus alumnos. La Educación Multicultural en los diferentes niveles de estudios debe entenderse como la educación que contribuye a generar el respeto y orgullo de las raíces

Ref. 31 Mire lo que yo quisiera decir es que este tipo de proyectos son de gran importancia nacional, ya que desglosan lo fue la cultura en esos tiempos y que nosotros debemos de enorgullecernos. Nosotros decimos ahora estamos en la "civilización", pero entendemos que, el tipo de hablantes, en esos tiempo remotos ya tenían adelantos sin tanta pompa como ahora, sin tanta licenciatura ya sabían de muchas cosas importantes que cualquiera diría, (...) Estos proyectos deberían de ser apoyados con mayor fuerza por el estado y con el propósito de impulsar como algo de conocimiento de la cultura que tenían nuestros antepasados antes de la conquista, un mayor apoyo es lo que necesita.
Profesor 07

C. E. Santo Domingo de Guzmán, Municipio Santo Domingo de Guzmán, Dpto. Sonsonate

indígenas del pueblo salvadoreño y que les permite su identificación en la actualidad. Transcendiendo esta formación hacia los educadores, para que puedan encontrar en las bases de la interculturalidad criterios que abonen a sus procesos educativos.

Así como lo expresa Fornet-Betancourt (2001), la interculturalidad no es un tema teórico sino principalmente una experiencia que no es producto de experiencias extraordinarias o excepcionales, sino que brota del ámbito común, del mundo cotidiano, en el sentido práctico que se está en contacto con el otro. Por tanto, la interculturalidad debe cultivarse de manera reflexiva y planificada para organizar las culturas. La interculturalidad es una cualidad que puede adquirir cualquier persona y cultura a partir de una praxis de vida en la que se cultive una comunicación-relación con el otro que trascienda la decodificación y que permita afectar, tocar, impresionar, tal como lo está haciendo el proyecto de revitalización del idioma náhuat.

\section{Referencias}

Banks, J. (2002). An Introduction to Multicultural Education. Boston: Pears.

Banks, J. (1996). Multicultural Education, Transformative Knowledge and Action. 1996. Teachers College Press NY en Banks, J. (Ed) The canon debate, Knowledge construction and multicultural education, 3-29. 
Corbin, J. y Strauss (2008). A Basics of Qualitative Research. USA: Sage.

Creswell, J. (2009). Research Design: Qualitative, Quantitative, and Mixed Mothods Approaches. USA: Sage.

Gadotti, M. (2003). Perspectivas actuales de la Educación. México D. F.: Editorial siglo XXI.

García-Castaño, J.; Pulido. R. y Montes, A. (1997). La ecuación multicultural y el concepto de cultura. Revista Iberoamerican de Educación 13, 223256.

Gregori, R. (2007, julio). Indígenas demandan al gobierno por discriminación racial en censo. Elfaro.net. Periódico digital latinoamericano. Consultado en http:// archivo. elfaro.net/secciones/el_agora/20070702/ElAgora1_20070702.asp

Fornet-Betancourt, R. (2001). Transformación intercultural de la filosofía. Bilbao: Desclée de Brouwer.

Lafaye, J. (1986). ¿ldentidad literaria o alteridad cultural? Identidad cultural de Iberoamérica en su literatura. 24-25.

Lara-Martínez, R. (2008). Balsamera bajo la guerra fría. El Salvador 1932. Historia de un etnocidio. San Salvador: Editorial Don Bosco.

LeCompte, M. D. and Schensul, J. J. (1999). Analyzing and interpreting ethnographic data. California: Sage.

Lemus, J. E. (2004). El pueblo pipil y su lengua. Científica 5, 7-28

Lemus, J. E. (201 1). Una aproximación a la definición del indígena salvadoreño. Científica 12, 3-17.

Lemus, J. E. (2008). Un modelo de revitalización lingüística: el caso del náhuat o pipil de El Salvador Diálogos 2, 48-61.

Meléndez, L. (2008). La Transformación Intercultural desde los Ambientes de Aprendizaje. XIV Congreso Internacional de Tecnología y Educación a Distancia. De la práctica educativa hacia la inclusión sociocultural. Universidad Estatal a Distancia. San José, Costa Rica.

Ministerio de Economía (2008). VI Censo de Población y V de Vivienda Población, Viviendas y Hogares 2007. El Salvador.

Naciones Unidas CERD (2005). Informes presentados por los estados partes de conformidad con el artículo 9 de la convención Decimoterceros informes periódicos que los Estados Partes debían presentar en 2004 Adición El Salvador. Convención Internacional sobre la eliminación de todas las Formas de Discriminación Racial. Tomado de http://www.bayefsky.com/reports/ elsalvador_cerd_c_471_ add_1_2005_sp.pdf

Requena, A.; Carrero, V.; Soriano, R. (2006). Teoría fundamentada."Grounded Theory. La construcción de la teória a través del análisis interpretacional. Madrid: CSI Cuadernos metodológicos

Ruiz Olabuenaga, J. I. e Ispizua, M. A. (1989). La entrevista enfocada. La descodificación de la vida cotidiana. Billbao: Universidad de Deusto.

Taylor, Ch. (1996). Identidad y reconocimiento. RIFP 7, 10-19. 